\title{
Autoimmune Pancreatitis (AIP) Masquerading as Pancreatic Cancer: Cutting Is Not a Crime ... for Now
}

\author{
Nicholas J. Zyromski
}

Published online: 26 June 2012

(C) Springer Science+Business Media, LLC 2012

Surgical decision-making in patients with a solid pancreatic mass is fairly straightforward: most pancreatic surgeons recommend resection. On the other hand, all series of pancreatoduodenectomy include a small percentage of patients resected for presumed malignancy who are ultimately found to have benign disease. A wide spectrum of rare conditions may masquerade as pancreatic adenocarcinoma; included in these conditions is autoimmune pancreatitis (AIP). Over the past decade, a great deal of attention has been directed toward cataloguing and better understanding the natural history of AIP. The condition is rare-large institutional series include less than a few 100 patients, and the Netherlands nationwide registry includes 130 patients to date. The diagnosis of AIP in some cases proves extremely challenging, leading to operative resection for presumed malignancy.

In this issue of Digestive Diseases and Sciences, the Erasmus University group (Rotterdam, the Netherlands) provides an excellent contemporary snapshot of patients found to have benign disease after undergoing pancreatoduodenectomy for presumed malignancy, specifically focusing on those found to have AIP [1]. Over a 10-year period, 23 of 274 (8\%) patients undergoing pancreatoduodenectomy for presumed malignancy were found to have benign disease. Of these 23 patients, nearly one-third had AIP $(n=6)$ or autoimmune cholangitis $(n=1)$. The index of suspicion for malignant disease was extremely high in the entire group of patients, who included those with very high circulating CA 19-9, double duct sign, and false positive fine needle aspiration pathology. So how do we

N. J. Zyromski (ه)

Department of Surgery, Indiana University School of Medicine, Indianapolis, IN, USA

e-mail: nzyromsk@iupui.edu avoid (or CAN we avoid) operating on patients with clinically suspected AIP?

The diagnosis of AIP is based on clinical suspicion, radiologic findings, and serologic [(immunoglobulin G4 (IgG4)] data. In some patients with equivocal findings but high suspicion for AIP, a short course of steroid treatment may be diagnostic as well as therapeutic. Clinical suspicion for AIP should be considered in patients with concomitant inflammatory or autoimmune disease. Certainly current awareness of AIP is elevated: the number of the authors' patients found to have AIP was halved in the latter period of their study. On the other hand, when evaluated retrospectively only one of seven patients would have been suspected to have AIP by applying two contemporary grading scores (HISORt and the Asian criteria). Serology is certainly not perfect, either. Circulating IgG4 concentration is elevated in some, but not all AIP patients. Of 20 patients treated at Indiana University with AIP confirmed by core or surgical biopsy, only one-third had elevated serum IgG4 [2]. The radiologic appearance of AIP is quite variable as well; none of these resected patients had the obvious classic "sausage" swelling of the pancreas. The only patient in the Dutch series documented to have received steroids was treated post-operatively (with spectacular response). In very select patients, a very short (2 week) trial of steroids may be indicated. This short duration will not delay definitive therapy if indeed the patient does prove to have a malignant process.

The Erasmus University report is timely; information about perioperative and long-term outcomes of AIP patients is emerging rapidly. Two very recent reports at this year's Pancreas Club meeting (19 May 2012) focused on just this topic (the full text of the Pancreas Club abstracts may be accessed at www.pancreasclub.com/final-program/ ). A combined series from the Mayo Clinic and 
Massachusetts General Hospital detailed 74 AIP patients resected over a 25 -year period, of whom $23 \%$ required post-operative steroid treatment; interestingly, many of these latter patients developed extrapancreatic inflammatory conditions, such as inflammatory bowel disease and immune thrombocytopenia purpura. The Indiana University group reported a case-matched series of ten patients with AIP resected for presumed malignancy over a contemporary 10-year period. No perioperative differences were noted in AIP patients compared to comparison groups resected for idiopathic chronic pancreatitis or pancreatic adenocarcinoma. Thirty percent of the AIP patients had documented disease recurrence either in the pancreas $(n=2)$ or the bile duct $(n=1)$. Therefore, even after resection of the most affected area, AIP patients deserve close follow-up.

So what is the take home message? Galloping hoof beats usually belong to horses, and not zebras. AIP is a rare condition. A 75-year-old smoker with painless jaundice due to a pancreatic head mass will most likely have pancreatic adenocarcinoma. However, in this day and age, even with heightened awareness of AIP and appropriate preoperative workup (including serum IgG4 measurement and, in very select cases, a short steroid trial), some patients with AIP are likely to be resected for the suspicion of malignancy. And that is all right-considering the alternative of not resecting a patient with operable pancreatic cancer. We will look forward to an improved understanding of AIP biology, which hopefully will translate into more accurate diagnosis methodology.

\section{References}

1. van Heerde MJ, Biermann K, Zondervan PE, et al. Prevalence of autoimmune pancreatitis and other benign disorders in pancreatoduodenectomy for presumed malignancy of the pancreatic head. Dig Dis Sci. 2012. (Epub ahead of print). doi:10.1007/s10620-0122191-7.

2. Moore SA, Cummings O, Sandrasegaran K, et al. Autoimmune pancreatitis in the Midwest US population: should we rely on elevated serum IgG4 for establishing the diagnosis? Am J Gastroenterol. 2008;103:S83. 